\title{
COVID-19-Associated Cytotoxic Lesions of the Corpus Callosum
}

\author{
(D) P. Gaur, (D) Dixon, (D) B. Jones, (D) H. Lyall, and (D). Jan
}

\section{ABSTRACT}

SUMMARY: There are very limited published data on the neurologic complications associated with coronavirus disease 2019 (COVID-19) in the pediatric population. Here we present the first 2 pediatric cases of presumed COVID-19 related cytotoxic lesions of the corpus callosum. Similar to reports in adults, these cases suggest that the COVID-19 infection in children may rarely mediate a hyperinflammatory response that can cause CNS pathology. As the pandemic continues further, the presentation of cytotoxic lesions of the corpus callosum should prompt radiologists to consider COVID-19, among other known causes.

ABBREVIATIONS: $\mathrm{CLOCC}=$ cytotoxic lesions of the corpus callosum; COVID-19 = coronavirus disease $2019 ; \mathrm{CRP}=\mathrm{C}$-reactive protein; RT-PCR = real-time reverse transcriptase polymerase chain reaction; SARS-CoV-2 = Severe Acute Respiratory Syndrome coronavirus 2

A of June 2, 2020, over 6.1 million cases of coronavirus disease 2019 (COVID-19) have been recorded and over 370,000 people have died. ${ }^{1}$ Most confirmed cases have been in the adult population with only a small percentage $(1 \%-2 \%)$ of reported cases being in children. ${ }^{2}$ In the pediatric population, COVID-19 infection seems to be often asymptomatic or associated with mild respiratory symptoms. ${ }^{2}$ As yet, there have been very limited reports of neurologic complications in children. Here we report the first series of pediatric neurologic presentations with COVID-19-associated cytotoxic lesions of the corpus callosum (CLOCC). This presentation has been related to other viral infections, but not yet demonstrated in association with COVID-19.

\section{CASE DETAILS}

A 12-year-old boy presented with a 5-day history of fever, lethargy, severe headache, vomiting, lower abdominal pain, and diarrhea. On examination, he was febrile and tender in the left iliac fossa and there was bilateral conjunctival infection, periorbital swelling, and scrotal edema. Neurologic examination was normal. Blood tests demonstrated lymphopenia and thrombocytopenia, with evidence of a systemic inflammatory response (raised D-dimer, fibrinogen, C-reactive protein [CRP], ferritin, lactate

Received June 3, 2020; accepted after revision June 11.

From the Departments of Imaging (P.G., L.D., B.J., W.J.) and Paediatric Infectious Diseases (H.L.), Imperial Healthcare NHS Trust, Charing Cross Hospital, London, United Kingdom.

Please address correspondence to Luke Dixon, Imaging Department, Charing Cross Hospital, Fulham Palace Rd, W6 8RF, London; e-mail: luke.dixon1@nhs.net

- Indicates open access to non-subscribers at www.ajnr.org

http://dx.doi.org/10.3174/ajnr.A6713

dehydrogenase, and procalcitonin). Urine and blood cultures were negative. Chest radiograph was indeterminate for COVID19. Real-time reverse transcriptase polymerase chain reaction (RT-PCR) analysis of 2 nasopharyngeal swabs was negative. A subsequent COVID-19 serology test, an immunoassay for the in vitro detection of IgG antibody specific to Severe Acute Respiratory Syndrome coronavirus 2 (SARS-CoV-2) in human serum and plasma, was positive. No other causative agent was isolated and serologic testing showed no evidence of other infection. In the context of the serology and supportive blood test results, a diagnosis of COVID-19 was reached. MR imaging of the brain, performed early into admission, showed a small, ovalshaped, T2-weighted hyperintense lesion in the splenium of the corpus callosum that exhibited restricted diffusion (Fig 1). There was no associated intrinsic susceptibility signal drop-out; postcontrast imaging was not performed. The patient was not on any medications or therapies that could have clearly accounted for the callosal lesion at the time of imaging. Shortly after admission he became hemodynamically shocked with raised troponin levels suggesting myocardial involvement. His level of consciousness and oxygen saturation worsened, without appreciable seizure phenomenon, necessitating intubation and mechanical ventilation. Based on the evidence of both cardiac and central nervous system pathology and blood tests supporting a hyperinflammatory response, a diagnosis of COVID-19-related pediatric multisystem inflammatory syndrome was made. ${ }^{3}$

The patient was treated with intravenous immunoglobulin, Anakinra (Interleukin-1 antagonist), infliximab, and steroids. The patient's condition improved rapidly soon after commencing therapy and there were no neurologic symptoms at discharge. 


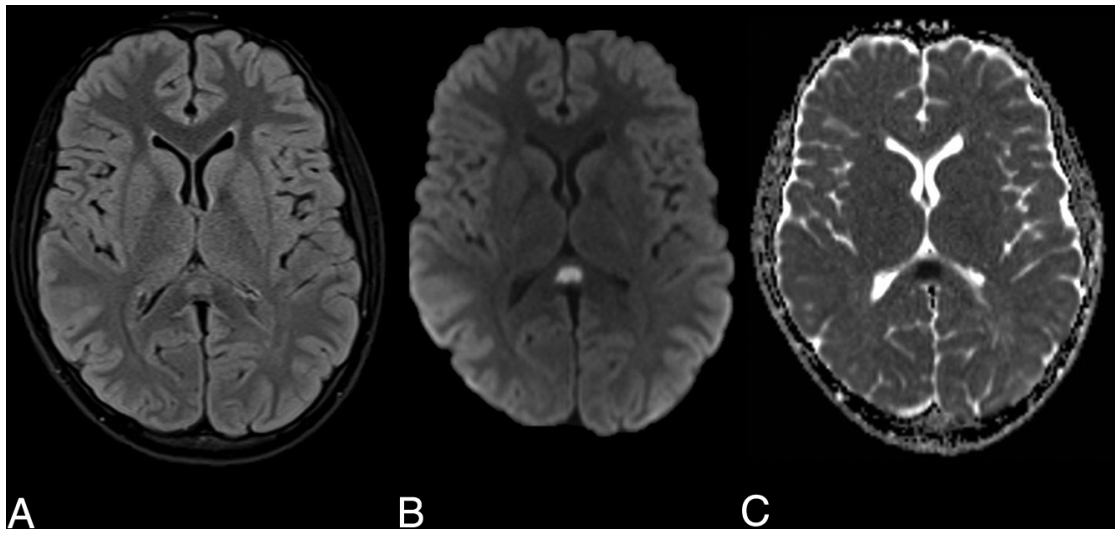

FIG 1. Axial MR images demonstrate an oval T2-weighted hyperintensity in the splenium of the corpus callosum $(A)$ with associated restricted diffusion on the DWI (B) and ADC maps (C).

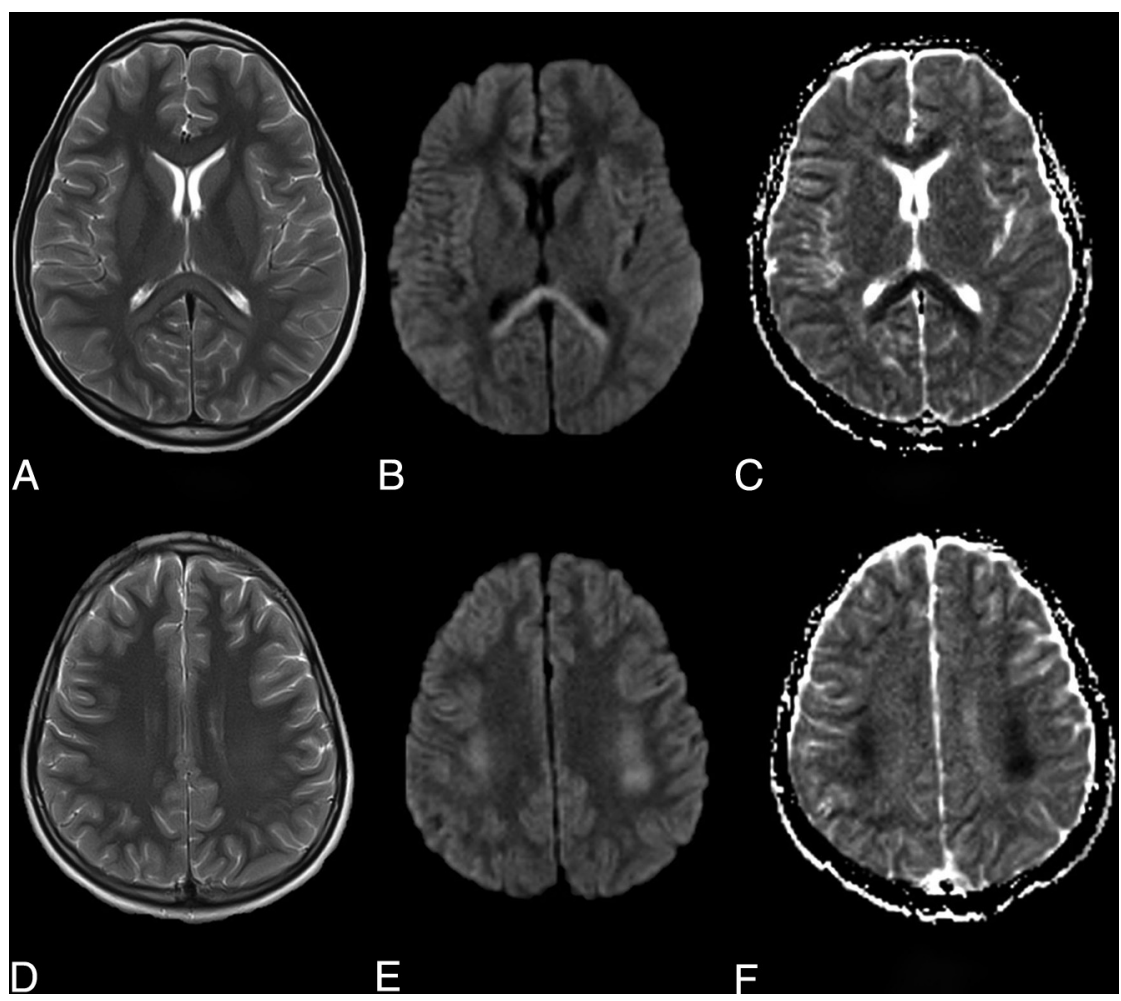

FIG 2. Axial MR images demonstrate T2-weighted hyperintensity in the splenium and genu of the corpus callosum $(A)$ and symmetrically in the deep cerebral white matter $(B)$ with associated restricted diffusion on the DWI $(B$ and $E)$ and $A D C$ maps $(C$ and $F)$.

Repeat MR imaging has not yet been performed and was felt unwarranted acutely due to the clinical resolution.

A second pediatric patient, a 9-year-old boy, presented with altered mental state, fever, and lethargy. On examination he was dysarthric and ataxic. Audible lung crepitations were noted on respiratory examination. Blood tests demonstrated a lymphopenia, raised C-reactive protein and serum ferritin. Urine and blood cultures were negative, and a chest radiograph was normal. An early head MR imaging showed similar but more extensive abnormal T2-weighted hyperintense signal and restricted diffusion in the entire corpus callosum and frontoparietal cerebral white matter.
This had a dorsal predilection, with greatest in-volvement of the splenium, peri-atrial white matter, and posterior frontal and parietal centrum semiovale (Fig 2). There was no intrinsic susceptibility signal drop-out. Postcontrast imaging was not performed. SARSCoV-2 on RT-PCR testing was subsequently confirmed on broncho-alveolar lavage, despite initial negative results from nasopharyngeal swabs. No other causative agent was isolated. This second patient's symptoms improved rapidly and early follow-up MR imaging showed almost complete resolution of abnormal signal. ${ }^{1}$

\section{DISCUSSION}

CLOCC is a clinical-radiologic syndrome with a wide range of reported causes, including infection, trauma, drug-related, metabolic disorders, and malignancy. ${ }^{4}$ It typically manifests in children and can present with reduced consciousness, nuchal rigidity, and seizures. ${ }^{2,4}$

CLOCC has been described in association with many pathogens, including influenza, malaria, Ebstein-Barr virus, adenovirus, and $E$ coli. ${ }^{5,6}$ In these cases where infection is thought to be the underlying cause, the diagnosis is also termed mild encephalitis/encephalopathy with a reversible splenial lesion. Our cases mirror clinical and radiologic features reported in a case series of 15 children with CLOCC of which $83 \%$ were presumed to be related to various different infections. Similar to our observations in presumptive COVID19 related CLOCC, the authors of that series demonstrated evidence of systemic inflammation (raised CRP) and different degrees of nonenhancing, diffusion-restricting, white matter signal abnormality from isolated splenial lesions to more diffuse corpus callosal and extracallosal involvement. ${ }^{6}$ To our knowledge no cases of CLOCC related to the inflammatory response to SARS-CoV-2 or other coronaviruses have been reported. CLOCC is thought to be caused by a cytokine storm that triggers $\mathrm{T}$ cells to breach the blood-brain barrier causing inflammation and intramyelinic edema. ${ }^{5,7}$ This aligns with our cases of CLOCC that appeared to occur in the context of a multisystem inflammatory process with one of the cases showing more classic signs of pediatric inflammatory multisystem syndrome temporally associated with SARS-CoV-2, including myocardial and abdominal involvement. ${ }^{3}$ These cases reflect a 
new presentation of CNS inflammatory complication of SARSCoV-2 exposure in children with pediatric inflammatory multisystem syndrome temporally associated with SARS-CoV-2. This inflammatory syndrome shares features with Kawasaki disease, toxic shock syndrome, and macrophage activation syndrome. $^{8-10}$ In our cases, the apparent rapid response to immunotherapy is encouraging and further supports the theory that this is an inflammatory and possibly cytokine-mediated process.

The diagnosis of CLOCC is based on the MR imaging demonstration of diffusion-restricting, nonenhancing lesions that usually resolve between a few days and weeks. Radiologically, as demonstrated in our cases, there is typically a lesion in the splenium of the corpus callosum with more variable involvement of the remaining corpus callosum and cerebral white matter. ${ }^{5}$ Often clinical recovery lags behind imaging, but is usually within 3 months. ${ }^{5}$

This is the first reported series of presumptive COVID-19associated CLOCC that appeared to occur in the context of pediatric inflammatory multisystem syndrome temporally associated with SARS-CoV-2. Further investigation of COVID-19-related CNS pathology in children and the potential role of immunotherapy is required. Clinicians and radiologists should be aware of this potential presentation.

\section{REFERENCES}

1. Geneva S. World Health Organization. Coronavirus disease 2019 (COVID-19) situation report - 105. World Health Organization https://www.who.int/docs/default-source/coronaviruse/situation-reports/ 20200504-covid-19-sitrep-105.pdf?sfvrsn=4cdda8af_2. Published 2020

2. Parri N, Lenge M, Buonsenso D. Children with Covid-19 in pediatric emergency departments in Italy. $N$ Engl J Med 2020;383:187-90 CrossRef Medline

3. Whittaker E, Bamford A, Kenny J, et al. Clinical characteristics of 58 children with a pediatric inflammatory multisystem syndrome temporally associated with SARS-CoV-2. JAMA 2020;324:259-69 CrossRef Medline

4. Takanashi JI. Two newly proposed infectious encephalitis/encephalopathy syndromes. Brain Dev 2009;31:521-28 CrossRef Medline

5. Vanderschueren G, Schotsmans K, Maréchal E, et al. Mild encephalitis with reversible splenial (MERS) lesion syndrome due to influenza B virus. Pract Neurol 2018;18:391-92 CrossRef Medline

6. Chen WX, Liu HS, Yang SD, et al. Reversible splenial lesion syndrome in children: Retrospective study and summary of case series. Brain Dev 2016;38:915-27 CrossRef Medline

7. Starkey J, Kobayashi N, Numaguchi Y, et al. Cytotoxic lesions of the corpus callosum that show restricted diffusion: mechanisms, causes, and manifestations. Radiographics 2017;37:562-76 CrossRef Medline

8. McGonagle D, Sharif K, O'Regan A, et al. The role of cytokines including interleukin-6 in COVID-19 induced pneumonia and macrophage activation syndrome-like disease. Autoimmun Rev 2020;19:102537 CrossRef Medline

9. Inciardi RM, Lupi L, Zaccone G, et al. Cardiac involvement in a patient with coronavirus disease 2019 (COVID-19). JAMA cardiology 2020 Mar 27. [Epub ahead of Print] CrossRef Medline

10. Jones VG, Mills M, Suarez D, et al. COVID-19 and Kawasaki disease: novel virus and novel case. Hosp Pediatr 2020;10:537-40 CrossRef Medline 